\title{
COVID-19 Is Not Over and Needs Prediction Scores: An Endless Road!
}

\author{
Ata Mahmoodpoor (iD ${ }^{1}$, Farnad Imani (iD) ${ }^{2}$ and Hassan Soleimanpour (iD) ${ }^{3,}{ }^{*}$ \\ ${ }^{1}$ Department of Anesthesiology and Critical Care Medicine, Faculty of Medicine, Tabriz University of Medical Sciences, Tabriz, Iran \\ ${ }^{2}$ Pain Research Center, Department of Anesthesiology and Pain Medicine, Iran University of Medical Sciences, Tehran, Iran \\ ${ }^{3}$ Emergency Medicine Research Team, Tabriz University of Medical Sciences, Tabriz, Iran \\ "Corresponding author: Emergency Medicine Research Team, Tabriz University of Medical Sciences, Tabriz, Iran. Email: h.soleimanpour@gmail.com, \\ soleimanpourh@tbzmed.ac.ir
}

Received 2021 December 03; Revised 2021 December 05; Accepted 2021 December 07.

Keywords: World Health Organization, SARS-CoV-2, Acute Respiratory Distress Syndrome, Intensive Care Unit, Mortality

Following the broad spread of SARS-CoV-2 in Wuhan City from December 2019, the World Health Organization (WHO) declared the novel coronavirus disease (COVID-19) a public health emergency of international concern; by 11 March 2020, the COVID-19 outbreak was declared a global pandemic (1, 2). Until now, no specific therapies have yet been developed to prevent or reduce the risk of developing complications and organ dysfunction of COVID-19 (3). Therefore, early and accurate identification of patients at higher risk of developing severe disease is so crucial (4) both as part of guidance for clinical treatment and resource allocation as well as to highlight the likely groups benefiting from novel disease-modifying therapies.

With the fast-changing pandemic landscape and emerging new information regarding therapeutic options, immune response, and virus mutations, it seems impossible that one prediction score can answer all questions. Many studies have been performed and shown the predictive value of different scores in multicenter studies, but their external clinical utility and face validity have not been established in independent cohorts $(5,6)$.

Recently, more than 20 specific scores for COVID-19 patients have been developed and introduced (7). Some of them are simple, but some are more complex systems using a combination of clinical and laboratory markers and comorbidities to predict the development of critical illness among hospitalized patients infected with COVID-19 $(8,9)$. acute physiology and chronic health evaluation (APACHE) is one of the most common prediction scores used in critically ill patients and is widely used as a predictor in critically ill patients. Karthick et al. showed that APACHE II could be used as a predicting score for hospital mortality in COVID-19 patients, especially when the score is more than 17. They emphasized that APACHE II was an early warning predictor of mortality and could guide physicians in conducting therapeutic protocol (10). The results of another study in COVID-19 showed that the APACHE II score was an effective clinical score for mortality prediction compared to the sequential organ failure assessment (SOFA) score and confusion, urea, respiratory rate, blood pressure, > 65 years of age (CURB-65) score (11).

As we know, the most common reason for mortality in COVID-19 patients is acute respiratory distress syndrome (ARDS). On the other hand, a definite dissociation between a clinically happy/silent hypoxemia and profound hypoxemic pulmonary failure is frequently seen. Thus, relying on this situation can misguide physicians regarding future intervention and deciding treatment protocols. The lung mechanic indices are well-preserved in the first days of the disease, and there is no increased airway resistance or dead space ventilation; thus, the respiratory center does not sense an uncomfortable sensation of breathing. Considering ARDS as the most common reason for mortality in COVID-19 patients and concurrent happy/silent hypoxemia on the first day of the disease, it seems that prediction scores that rely on pulmonary variables (such as partial pressure of oxygen $\left[\mathrm{PaO}_{2}\right], \mathrm{PaO}_{2} /$ fraction of inspired oxygen $\left[\mathrm{FiO}_{2}\right]$ ) can be misleading in diagnosing the severe/critically ill COVID-19 patients (12-15).

The problem arises when these scores are used to predict long-term mortality, especially after intensive care unit (ICU) discharge. The mortality of COVID-2019 patients was higher than that previously seen in critically ill patients for some reasons as follows: First, a large number of COVID-19 cases that occurred in a short period led to difficulty in hospitalization treatment. Second, the lack of information regarding clinical characteristics and pathophysiological features of the disease. Third, the APACHE II score was assessed by the characteristics of the patients on the first day of ICU admission, which, based on the mentioned comments regarding pulmonary involvement and happy hypoxemia, can be misinterpreted as normal 
or near-normal values. Forth, regarding the possibility of adhering to some national guidelines of "stay at home," patients were referred to hospitals with more severe disease, which made their management difficult. Based on big data and problems regarding COVID-19 management, Ko et al. developed an artificial intelligence model using blood biomarkers at the time of hospital admission for mortality prediction in COVID-19 patients with an accurate validity (16).

Finally, considering the fact that mortality prediction is neither perfect nor absolute, it seems that considering clinical symptoms of patients and increasing the level of standard of care and healthcare workers' information are the most important points for the best treatment strategy and decreasing the mortality of COVID-19 patients. However, using a simple bedside score to predict the possibility of severe disease/mortality of COVID-19 patients can help physicians regarding their treatment options. We recommend future studies with adequate inclusion/censoring and description of the study population, defining the time horizon of the prediction, and structured and standard reporting of results (17); however, they need external validation and other studies to prove their usefulness.

\section{Acknowledgments}

We would like to appreciate the cooperation of the Clinical Research Development Unit, Imam Reza General Hospital, Tabriz, Iran, in conducting this research.

\section{Footnotes}

Authors' Contribution: Study concept and design: H. S., F. I., and A. M.; Drafting of the manuscript: A. M., F. I., and H. S. All authors read and approved the final manuscript. All authors reviewed the final manuscript.

Conflict of Interests: The authors declare no conflict of interest.

\section{References}

1. Mahmoodpoor A, Shadvar K, Ghamari AA, Mohammadzadeh Lameh M, Asghari Ardebili R, Hamidi M, et al. Management of Critically Ill Patients with COVID-19: What We Learned and What We Do. Anesth Pain Med. 2020;10(3). e104900. doi: 10.5812/aapm.104900. [PubMed: 32944565]. [PubMed Central: PMC7472789].

2. Bagi HM, Soleimanpour M, Abdollahi F, Soleimanpour H. Evaluation of clinical outcomes of patients with mild symptoms of coronavirus disease 2019 (COVID-19) discharged from the emergency department. PLoS One. 2021;16(10). e0258697. doi: 10.1371/journal.pone.0258697. [PubMed: 34673806]. [PubMed Central: PMC8530279].

3. Pergolizzi JJ, Magnusson P, LeQuang JA, Breve F, Paladini A, Rekatsina $\mathrm{M}$, et al. The Current Clinically Relevant Findings on COVID-19 Pandemic. Anesth Pain Med. 2020;10(2). e103819. doi: 10.5812/aapm.103819. [PubMed: 32754437]. [PubMed Central: PMC7352949].
4. Rahimzadeh P, Amniati S, Farahmandrad R, Faiz SHR, Hedayati Emami S, Habibi A. Clinical Characteristics of Critically Ill Patients Infected with COVID-19 in Rasoul Akram Hospital in Iran: A Single Center Study. Anesth Pain Med. 2020;10(5). e107211. doi: 10.5812/aapm.107211. [PubMed: 34150569]. [PubMed Central: PMC8207836].

5. Wynants L, Van Calster B, Collins GS, Riley RD, Heinze G, Schuit E, et al. Prediction models for diagnosis and prognosis of covid19: Systematic review and critical appraisal. BMJ. 2020;369:m1328. doi: 10.1136/bmj.m1328. [PubMed: 32265220]. [PubMed Central: PMC7222643].

6. Sanaie S, Hosseini MS, Karrubi F, Iranpour A, Mahmoodpoor A. Impact of Body Mass Index on the Mortality of Critically Ill Patients Admitted to the Intensive Care Unit: An Observational Study. Anesth Pain Med. 2021;11(1). e108561. doi: 10.5812/aapm.108561. [PubMed: 34249664]. [PubMed Central: PMC8256440].

7. Martinez-Lacalzada M, Adrián Viteri-Noël L, Manzano L, FabregateFuente M, Rubio-Rivas M, Garcia SL, et al. Predicting critical illness on initial diagnosis of COVID-19: development and validation of the PRIORITY model for outpatient applicability. MedRxiv. 2020. doi: 10.1101/2020.11.27.20237966.

8. Guo L, Wei D, Zhang X, Wu Y, Li Q, Zhou M, et al. Clinical Features Predicting Mortality Risk in Patients With Viral Pneumonia: The MuLBSTA Score. Front Microbiol. 2019;10:2752. doi:10.3389/fmicb.2019.02752. [PubMed: 31849894]. [PubMed Central: PMC6901688].

9. Liang W, Liang H, Ou L, Chen B, Chen A, Li C, et al. Development and Validation of a Clinical Risk Score to Predict the Occurrence of Critical Illness in Hospitalized Patients With COVID-19. JAMA Intern Med. 2020;180(8):1081-9. doi:10.1001/jamainternmed.2020.2033. [PubMed: 32396163]. [PubMed Central: PMC7218676].

10. Karthick D, Divahar M, Rajmohan S, Jayalal JA. Apache II Score as a Predictor of Hospital Mortality in COVID-19 Patients. Int J Surg Res. 2020;9(1):9-16.

11. Zou X, Li S, Fang M, Hu M, Bian Y, Ling J, et al. Acute Physiology and Chronic Health Evaluation II Score as a Predictor of Hospital Mortality in Patients of Coronavirus Disease 2019. Crit Care Med.2020;48(8):e657-65. doi:10.1097/CCM.0000000000004411. [PubMed: 32697506]. [PubMed Central: PMC7217128].

12. Haryalchi K, Heidarzadeh A, Abedinzade M, Olangian-Tehrani S, Ghaz anfar Tehran S. The Importance of Happy Hypoxemia in COVID-19. Anesth Pain Med. 2021;11(1). e111872. doi: 10.5812/aapm.111872. [PubMed: 34221943]. [PubMed Central: PMC8239260].

13. Kafan S, Tadbir Vajargah K, Sheikhvatan M, Tabrizi G, Salimzadeh A, Montazeri M, et al. Predicting Risk Score for Mechanical Ventilation in Hospitalized Adult Patients Suffering from COVID-19. Anesth Pain Med.2021;11(2). e112424. doi:10.5812/aapm.112424. [PubMed: 34336617]. [PubMed Central: PMC8314086].

14. Sanaie S, Mahmoodpoor A, Hamishehkar H, Shadvar K, Salimi N, Montazer M, et al. Association Between Disease Severity and Calcium Concentration in Critically Ill Patients Admitted to Intensive Care Unit Anesth Pain Med. 2018;8(1). e57583. doi: 10.5812/aapm.57583. [PubMed: 29868455]. [PubMed Central: PMC5970362].

15. Olapour A, Rashidi M, Javaher Foroush F, Akhoondzadeh R, Hosseini N. The Effect of Nebulized Heparin on Weaning off Intubated Patients with Acute Respiratory Distress Syndrome (ARDS) Admitted to Intensive Care Unit (ICU): A Randomized Clinical Trial. Anesth Pain Med. 2021;11(5). e115938. doi: 10.5812/aapm.115938.

16. Ko H, Chung H, Kang WS, Park C, Kim DW, Kim SE, et al. An Artificial Intelligence Model to Predict the Mortality of COVID-19 Patients at Hospital Admission Time Using Routine Blood Samples: Development and Validation of an Ensemble Model. J Med Internet Res. 2020;22(12). e25442. doi: 10.2196/25442. [PubMed: 33301414]. [PubMed Central: PMC7759509].

17. Collins GS, Reitsma JB, Altman DG, Moons KG. Transparent Reporting of a multivariable prediction model for Individual Prognosis or Diagnosis (TRIPOD): the TRIPOD statement. Ann Intern Med. 2015;162(1):5563. doi: 10.7326/M14-0697. [PubMed: 25560714]. 